\title{
Identification of Lynch syndrome by microsatellite instability and mismatch repair deficiency testing on colorectal adenomas
}

\author{
Leah H. Biller ${ }^{1}$ Matthew B. Yurgelun ${ }^{1}$
}

Received: 27 November 2019 / Accepted: 3 December 2019 / Published online: 19 December 2019

(c) European Society of Human Genetics 2019

In a forthcoming issue, Dabir et al. present the results of a systematic review and meta-analysis, demonstrating the utility of using mismatch repair deficient (dMMR) and/or microsatellite instability (MSI) testing of colorectal adenomatous polyps to identify individuals with Lynch syndrome (LS) [1]. LS, caused by autosomal dominant inheritance of deleterious germline variants in the DNA mismatch repair (MMR) genes (MLH1, MSH2, MSH6, and PMS2) or EPCAM, is one of the most common forms of hereditary cancer predisposition, with an estimated prevalence of more than 1 in 300 individuals in the general population, the vast majority of whom are undiagnosed [2]. Early and frequent colonoscopic screening is known to improve overall mortality in LS, while aspirin chemoprevention and riskreducing gynecologic surgery can reduce incidence of LSassociated colorectal cancer (CRC) and endometrial cancer (EC), respectively, thus highlighting the critical importance of identifying LS carriers [3]. The classic method by which LS carriers are recognized is by testing CRC or EC specimens for the presence of dMMR and/or MSI, and numerous professional societies recommend that all CRC specimens undergo such testing as a screen for LS, due to its high sensitivity (89.7-91.4\%) and specificity (94.6-94.8\%) [4]. The main pitfall of this classic tumor testing strategy, however, is that it inherently relies on the development of an invasive cancer, and thus the opportunity for primary cancer prevention has already been missed.

The study by Dabir et al. thus systematically analyzed the available literature to explore the question of whether or not dMMR/MSI testing of colorectal adenomatous polyps (adenomas) can effectively identify LS, thereby potentially facilitating detection of carriers before the

Matthew B. Yurgelun

matthew_yurgelun@dfci.harvard.edu

1 Dana-Farber Cancer Institute, Brigham \& Women's Hospital, Harvard Medical School, Boston, MA, USA development of cancer [1]. The investigators analyzed 41 published studies, inclusive of 1142 adenomas from over 519 LS carriers and 2213 adenomas from 1698 individuals without known LS. In aggregate, they found that dMMR/ MSI was present in $69.5 \%$ of adenomas from LS carriers compared with only $2.8 \%$ among those without known LS. In LS carriers, adenomas with villous histology, those $>1$ $\mathrm{cm}$ in size, those with high-grade dysplasia, and those from individuals $>60$ years in age were significantly more likely to demonstrate dMMR/MSI (>80\% sensitivity for each such feature) compared with those lacking such features. Neither anatomic adenoma location within the large bowel nor specific MMR gene involved were significantly associated with dMMR/MSI status in adenomas from LS carriers.

These findings provide compelling evidence for the use of dMMR/MSI testing of colorectal adenomas as a viable strategy for identifying LS carriers, which leads naturally to the question of whether such testing could be applied to adenomas found during general population colonoscopic CRC screening as a means of detecting LS. In addition to the near $70 \%$ sensitivity, these data indicate that the presence of dMMR/MSI in a colorectal adenoma is at least $97 \%$ specific for a diagnosis of LS (this specificity would be even higher if any of the $2.8 \%$ of "sporadic" adenomas with $\mathrm{dMMR} / \mathrm{MSI}$ were actually from individuals with as yet unrecognized LS) [1].

While such statistics are certainly intriguing for systematic general population LS screening in colorectal adenomas, several important questions remain unanswered. Most national guidelines recommend initiation of CRC screening at age 50, yet data from the Prospective Lynch Syndrome Database have shown that up to $41.5 \%$ of LS carriers will have had a cancer diagnosis by age 50 , suggesting that many as yet unidentified LS carriers will develop cancer before there is even an opportunity to detect a large bowel adenoma on routine colonoscopic screening and perform testing for dMMR/MSI [5]. Recent preliminary data have also intriguingly suggested that testing normal colonic mucosa for dMMR/MSI may be an alternative 
means of identifying some LS carriers, since a small fraction of histologically nonneoplastic intestinal crypts will demonstrate dMMR/MSI in LS carriers but not normal controls [6]. The cost effectiveness of using dMMR/MSI in noncancerous large bowel tissue remains unknown, and it would be important to compare the efficacy and cost effectiveness of any such population-wide molecular screening with low-cost LS screening methods such as prediction modeling with PREMM5 (http://premm.dfci.ha rvard.edu), which requires only basic personal and family history data in order to generate a numeric likelihood of underlying LS. It is also worth cautioning that the potential utility of dMMR/MSI as a screen for LS applies only to adenomatous polyps and not to serrated polyps/adenomas of the large bowel. Serrated polyps/adenomas are the precursor lesions to most sporadic, non-LS-associated dMMR/MSI CRCs (which tend to occur predominantly in older individuals), and these serrated polyps/adenomas are known to commonly have acquired hypermethylation of the $M L H 1$ promoter with absent MLH1 expression by IHC, particularly in the presence of dysplasia. Thus, the presence of dMMR/MSI in serrated polyps/adenomas of the large bowel should not be considered compelling evidence of underlying LS.

In summary, numerous prior small reports have hinted at the potential utility of dMMR/MSI molecular testing on large bowel adenomas to identify LS, but the impact of such studies has been limited by their small sample size. Through systematic review and meta-analysis of this body of literature, Dabir et al. now provide robust data demonstrating that dMMR/MSI testing of adenomatous polyps indeed has an exceedingly high specificity and moderate sensitivity for underlying LS, particularly among larger adenomas, those with villous histology and/or high-grade dysplasia, and those found in older individuals [1]. Given the preventability of LS-associated CRC and EC with colonoscopies, aspirin, and risk-reducing hysterectomy, data such as these that further expand the methods by which clinicians can identify LS carriers, especially before the onset of cancer, are critical to realizing the goal of genetically driven cancer prevention.

\section{Compliance with ethical standards}

Conflict of interest The authors declare that they have no conflict of interest.

Publisher's note Springer Nature remains neutral with regard to jurisdictional claims in published maps and institutional affiliations.

\section{References}

1. Dabir, PD, Bruggeling CE, van der Post RS, Dutilh BE, Hoogerbrugge N, Ligtenberg MJL, et al. Microsatellite instability screening in colorectal adenomas to detect Lynch syndrome patients? A systematic review and meta-analysis. Eur J Hum Genet. 2019. https://doi.org/10.1038/s41431-019-0538-7

2. Win AK, Jenkins MA, Dowty JG, Antoniou AC, Lee A, Giles GG, et al. Prevalence and penetrance of major genes and polygenes for colorectal cancer. Cancer Epidemiol Biomark Prev. 2017;26: 404-12.

3. Boland PM, Yurgelun MB, Boland CR. Recent progress in Lynch syndrome and other familial colorectal cancer syndromes. CA Cancer J Clin. 2018;68:217-31.

4. Hampel H, Pearlman R, Beightol M, Zhao W, Jones D, Frankel WL, et al. Assessment of tumor sequencing as a replacement for Lynch syndrome screening and current molecular tests for patients with colorectal cancer. JAMA Oncol. 2018;4:806-13.

5. Dominguez-Valentin, M, Sampson JR, Seppälä TT, Ten Broeke SW, Plazzer JP Nakken S, et al. Cancer risks by gene, age, and gender in 6350 carriers of pathogenic mismatch repair variants: findings from the Prospective Lynch Syndrome Database. Genet Med. 2019.

6. Pai RK, Dudley B, Karloski E, Brand RE, O'Callaghan N, Rosty C, et al. DNA mismatch repair protein deficient non-neoplastic colonic crypts: a novel indicator of Lynch syndrome. Mod Pathol. 2018;31:1608-1618. 